Preprint typeset in JHEP style - HYPER VERSION

SUBATECH 2005/004

LAPTH-1110/05

\title{
Retardation Effect for Collisional Energy Loss of Hard Partons Produced in a QGP
}

\author{
Stéphane Peigné; Pol-Bernard Gossiaux, Thierry Gousset \\ SUBATECH, UMR 645\%, Université de Nantes \\ Ecole des Mines de Nantes, IN2P3/CNRS. \\ 4 rue Alfred Kastler, 44307 Nantes cedex 3, France \\ E-mail: Iastname@subatech.in2p3.fr
}

\begin{abstract}
We study the collisional energy loss suffered by an energetic parton travelling the distance $L$ in a high temperature quark-gluon plasma and initially produced in the medium. We find that the medium-induced collisional loss $-\Delta E(L)$ is strongly suppressed compared to previous estimates which assumed the collisional energy loss rate $-d E / d x$ to be constant. The large $L$ linear asymptotic behaviour of $-\Delta E(L)$ sets in only after a quite large retardation time. The suppression of $-\Delta E(L)$ is partly due to the fact that gluon bremsstrahlung arising from the initial acceleration of the energetic parton is reduced in the medium compared to vacuum. The latter radiation spectrum is sensitive to the plasmon modes of the quark-gluon plasma and has a rich angular structure.
\end{abstract}

KeYwords: Quark Gluon Plasma, Collisional Energy Loss, Induced Radiation.

\footnotetext{
${ }^{*}$ On leave of absence from LAPTH, CNRS, UMR 5108, Université de Savoie, B.P. 110, F-74941 Annecyle-Vieux Cedex, France
} 


\section{Introduction}

Jet quenching has long ago been suggested as a possible signal for the quark-gluon plasma (QGP) [1], and this has triggered a considerable activity both on the experimental and theoretical sides. The theoretical determination of parton energy loss has been the subject of many studies, and numerous observables sensitive to jet quenching are currently analyzed in the Relativistic Heavy Ion Collider (RHIC) experiments.

In a (static) thermal or dense medium of very large size $L$, a parton of high (but finite) energy $E$ undergoes a radiative energy loss $\Delta E_{\text {rad }}$ which increases as a power of the energy $\left(\Delta E_{\text {rad }} \propto L E\right.$ in the Bethe-Heitler limit and $\Delta E_{\text {rad }} \propto L \sqrt{E}$ when the non-abelian LPM effect is at work [2]), whereas its collisional energy loss $\Delta E_{\text {coll }}$ behaves at most logarithmically $^{1}, \Delta E_{\text {coll }} \propto L \log E\left[1\right.$, 3, 4]. For a large size medium we thus expect $\Delta E_{\text {rad }}$ to be the dominant source of energy loss for energetic partons.

In the opposite limit, namely for a parton of asymptotic energy $E \rightarrow \infty$ crossing a medium of finite size $L<L_{c r}$ (but still thick enough, $L / \lambda \gg 1$, where $\lambda$ is the parton mean free path), $\Delta E_{\text {rad }}$ becomes independent of $E, \Delta E_{\text {rad }} \propto E^{0} L^{2}$ [6, ]]. The finite size limit $L<L_{c r}$ should be relevant in practice since a simple numerical estimate within the model of Ref. [6] gives $L_{c r}=\sqrt{\lambda E / m_{D}^{2}} \simeq 7 \mathrm{fm}$ for $E=10 \mathrm{GeV}$, where $m_{D}^{-1}$ is the Debye screening length in the medium. It was also shown [8] that finite opacity $(L / \lambda \gtrsim 1)$ and finite $E$ effects lead to a substantial suppression (together with some energy dependence) of $\Delta E_{\text {rad }}$, when compared to the asymptotic $E$-independent result. We can thus expect $\Delta E_{\text {coll }}$ to compete with $\Delta E_{\text {rad }}$ in the case of a finite size medium. Indeed, recent studies [9, 10, 11] suggest that for 'jets' of energy on the order of $10 \mathrm{GeV}$ such as those measured in $A A$ collisions at RHIC, the collisional energy loss might be comparable to the radiative one, both for light [10] and heavy [11] partons.

Thus an accurate determination of $\Delta E_{\text {coll }}$ for large $E$ and finite $L$ is needed in order to interpret the suppression of inclusive large $p_{T}$ hadron production at moderate energies observed at RHIC in $A A$ collisions [12, 13]. In previous studies of parton collisional energy loss in a QGP, the $\operatorname{loss}^{2}-\Delta E(L)$ suffered by the hard parton travelling the distance $L$ in the plasma was assumed to be given by $(-d E / d x)_{\infty}$ times $L$, with $(-d E / d x)_{\infty}$ the rate of energy loss per unit distance occurring in a stationary regime, i.e., long after the energetic parton has been produced. This is certainly a valid approximation when the medium size becomes very large, and formulas for $(-d E / d x)_{\infty}$ of a heavy quark of mass

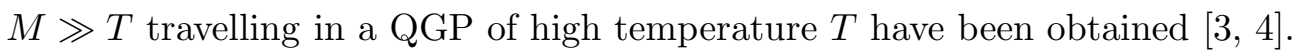

In the present paper we argue that such an approximation to $-\Delta E(L)$ is incorrect in the case of an energetic parton produced at an initial time $t=0$ inside a thermal or dense medium of moderate size $L$, which is the relevant situation when discussing jet or hadron quenching at large $p_{T}$. As in previous studies of collisional energy loss [3, 4], we work in the theoretical high temperature small coupling limit $g \ll 1$ for the QGP (and in the hard thermal loop - HTL - resummation framework 14, 15), and assume a fixed

\footnotetext{
${ }^{1}$ The $\log E$ factor arises when $\Delta E_{\text {coll }}$ is calculated in the fixed coupling approximation [1, 3, ,4, but is expected to be absent in the case of a running coupling [e]. This point is briefly discussed in section 3.2.

${ }^{2}$ From now on all energy losses will be implicitly collisional, $-\Delta E \equiv-\Delta E_{\text {coll }}$.
} 
coupling $\alpha_{s}$. We also consider a static (non-expanding) QGP in thermal equilibrium. As argued in section 3.2, we expect the retardation of the stationary regime found in this paper to be qualitatively unchanged in the case of a running coupling. However, a rigorous treatment taking into account the running of $\alpha_{s}$ would be needed in order to obtain a reliable quantitative estimate of $-\Delta E(L)$. Recalling moreover that $g\left(m_{D}\right) \gtrsim 1$ in realistic phenomenological applications, we stress that our results should be considered on a qualitative level only.

For a fast parton prepared at $t=-\infty$ and travelling in an infinite medium, the collisional energy loss can be understood as follows. The proper (chromo-)electric field of the parton polarizes the medium, which creates an effective (medium-induced) electric field around the parton, responsible for its slowing down. If the energetic parton is produced at $t=0$ (via some process involving a hard scale $\sim p_{T}$ ), we may expect the rate of collisional energy loss $-d E / d x$ to be reduced during the time the parton proper field is created, before reaching the asymptotic value $(-d E / d x)_{\infty}$. A result suggestive of such a retardation effect is qualitatively what we find in the following. A fast parton produced initially in the medium needs to travel some distance before losing energy at the highest rate. Our main conclusion is that collisional losses used in the analysis of nuclear modification factors $R_{A A}$ at large $p_{T}$ (for jet or hadron production) have been systematically overestimated due to the neglect of this effect.

In section 2 we present our model for the induced collisional energy loss $-\Delta E(L)$ of a parton produced initially in a QGP. In section 3 we give our numerical results for the $L$-dependence of $-\Delta E(L)$, which display a strong attenuation of the energy loss when compared to previous estimates, as well as a large retardation time $t_{\text {ret }}$ of the stationary regime. We give a simple interpretation of the largeness of $t_{\text {ret }}$. We also discuss the domain of validity of our analysis, and how the running of $\alpha_{s}$ could affect $-\Delta E(L)$. Finally, in section 4 , we show that within our (standard) definition of collisional energy loss, $-\Delta E(L)$ gets a contribution from induced gluon radiation which arises from the sudden acceleration of the parton at $t=0$. The in-medium bremsstrahlung spectrum due to charge acceleration is indeed not the same as in vacuum since it is sensitive to the longitudinal and transverse plasmon modes of the QGP. The angular spectrum presents a diffraction pattern depending on the plasma size $L$. The induced radiated energy is negative, and thus contributes (but only partly) to the suppression of $-\Delta E(L)$ and to the retardation effect discussed in section 3. We conclude in section 5 .

\section{Model for collisional energy loss of a parton produced at $t=0$ in a QGP}

We derive in this section the master equation (2.9) for the collisional energy loss $-\Delta E(L)$ of an energetic (and massive) parton of velocity $v$, initially produced in a quark-gluon plasma, and travelling the distance $L$ in the medium. We first present our model for the classical

partonic current density $j^{\mu a}=\left(\rho^{a}, \vec{j}^{a}\right)$, and then give, in the abelian approximation, the expression of the (chromo-)electric field induced by this current density. 


\subsection{Model for partonic current}

In previous studies of parton collisional energy loss in a QGP [3, 田, the classical color charge has been assumed to be produced at $t=-\infty$. In this case the current 4 -vector in coordinate space reads:

$$
j_{\infty}^{\mu a}(t, \vec{x})=q^{a} V^{\mu} \delta^{3}(\vec{x}-\vec{v} t),
$$

where $V^{\mu}$ denotes the parton 4 -velocity, $V=(1, \vec{v})$. The color index $a$ is carried by the parton color charge $q^{a}$ defined by $q^{a} q^{a}=C_{R} \alpha_{s}$, where $C_{R}$ is the Casimir invariant of the color representation $R$ the parton belongs to $\left(C_{R}=C_{F}=4 / 3\right.$ for a quark and $C_{R}=C_{A}=3$ for a gluon). In 4 -momentum space $K=(\omega, \vec{k})$ the current (2.1) becomes

$$
j_{\infty}^{\mu a}(K)=2 \pi q^{a} V^{\mu} \delta(K . V),
$$

which trivially satisfies current conservation, $K \cdot j_{\infty}=0$.

When considering the quenching of large $p_{T}$ jets or hadrons in relativistic heavy ion collisions, the large $p_{T}$ parent parton is rather created at $t=0$ in the underlying hard partonic subprocess. Its associated classical 'current' is thus, instead of (2.1),

$$
j_{0}^{\mu a}(t, \vec{x})=q^{a} V^{\mu} \delta^{3}(\vec{x}-\vec{v} t) \theta(t),
$$

giving in momentum space:

$$
j_{0}^{\mu a}(K)=i q^{a} \frac{V^{\mu}}{K . V+i \eta}=q^{a} V^{\mu}\left[i \mathrm{P}\left(\frac{1}{K . V}\right)+\pi \delta(K . V)\right] .
$$

However, the 'current' (2.4) is not conserved, $K . j_{0} \neq 0$. Color charge conservation requires the hard parton to be produced in conjunction with at least another parton in the partonic subprocess. As a generic conserved partonic current, we will consider the simple case of a (color singlet) dipole produced at $t=0$, consisting of two partons (with the same color charge $\left.q^{a}\right)$ of 4 -velocities $V_{1}=\left(1, \vec{v}_{1}\right)$ and $V_{2}=\left(1, \vec{v}_{2}\right)$ :

$$
j^{\mu a}=\left(\rho^{a}, \vec{j}^{a}\right)=i q^{a}\left(\frac{V_{1}^{\mu}}{K . V_{1}+i \eta}-\frac{V_{2}^{\mu}}{K . V_{2}+i \eta}\right) .
$$

In the following we will consider the second parton to be a static heavy quark, i.e. $\vec{v}_{2}=\overrightarrow{0}$, in which case the space component of the current is simply given by the first term of (2.5). The choice (2.5) instead of (2.4) is however crucial. Current conservation indeed constrains the form of the electric field (2.6), and allows to treat unambiguously the potential singularity at $\omega=0$ (see (2.6) and (2.7)), as we briefly explain below. We expect the main results of our study not to depend on the simplifying assumption $\vec{v}_{2}=\overrightarrow{0}$.

\subsection{Induced electric field}

Following [3] (see also [16]), in the abelian approximation and within linear response theory the Maxwell equations can be solved in 4-momentum space $K=(\omega, \vec{k})$, yielding the total (chromo-)electric field $\vec{E}^{a}$ in terms of the classical vector current density $\vec{j}^{a}$ :

$$
\epsilon_{L} \vec{E}_{L}^{a}+\left(\epsilon_{T}-k^{2} / \omega^{2}\right) \vec{E}_{T}^{a}=\frac{4 \pi}{i \omega}\left(\vec{j}_{L}^{a}+\vec{j}_{T}^{a}\right) .
$$


The longitudinal and transverse components are given by $\vec{j}_{L}=\left(\vec{j} \cdot \vec{k} / k^{2}\right) \vec{k}$ (we denote $k=$ $|\vec{k}|)$ and $\vec{j}_{T}=\vec{j}-\vec{j}_{L}$. In the linear response approximation the longitudinal and transverse dielectric functions of the plasma $\epsilon_{L}$ and $\epsilon_{T}$ are not affected by the external current. We consider a high temperature QGP, for which $\epsilon_{L, T}$ have been obtained in [17, 18] and later rederived in the gauge-invariant HTL resummation framework [14, 15].

In the following we will have to deal with the $1 / \omega$ potential singularity appearing in (2.6), and specifically affecting the longitudinal part of the electric field. The latter actually arises from Coulomb's law $k \epsilon_{L} E_{L} \propto \rho$, where $\rho$ is the charge density, by using the equation for current conservation $\rho=k j_{L} / \omega$. More precisely, our conserved current (2.5) satisfies $\rho=k j_{L} /(\omega+i \eta)$, for any $\vec{v}_{1}$ and $\vec{v}_{2}$ (including $\vec{v}_{2}=\overrightarrow{0}$ ). This shows that the potential $1 / \omega$ singularity appearing in (2.6) should be regularized with the retarded prescription, $\omega \rightarrow \omega+i \eta$.

We obtain from (2.6) the medium-induced electric field $\overrightarrow{\mathcal{E}^{a}}$,

$$
\overrightarrow{\mathcal{E}}^{a}(t, \vec{x})=\int_{-\infty}^{\infty} \frac{d \omega}{\omega} \int \frac{d^{3} \vec{k}}{4 \pi^{3} i} e^{-i(\omega t-\vec{k} \cdot \vec{x})}\left[\frac{\vec{j}_{L}^{a}}{\epsilon_{L}}+\frac{\vec{j}_{T}^{a}}{\epsilon_{T}-k^{2} / \omega^{2}}\right]_{\mathrm{ind}},
$$

where $\vec{j}^{a}$ is given by (2.5) (with $\vec{v}_{2}=\overrightarrow{0}$ ), or equivalently by (2.4) (with $\vec{v}=\vec{v}_{1}$ ), and the $1 / \omega$ singularity should be treated with the retarded prescription. In (2.7) the subscript denotes the implicit subtraction of the vacuum contribution (corresponding to $\epsilon_{L}=\epsilon_{T}=1$ ). Since the dielectric functions (and the external current) are real quantities in coordinate space, implying in momentum space $\epsilon_{L, T}(-K)=\epsilon_{L, T}(K)^{*}$ (and a similar relation for the current), the expression (2.7) is easily checked to be also real.

\subsection{Master equation for parton collisional energy loss}

During its travel in the plasma between $t=0$ and $t=L / v$, the induced energy gain $\Delta E$ of the parton of constant velocity $\vec{v}_{1}=\vec{v}$ equals the work of the electric force on its trajectory, namely $\Delta E=\vec{v} \cdot \int_{0}^{L / v} d t q^{a} \overrightarrow{\mathcal{E}}^{a}(t, \vec{x}=\vec{v} t)$ or:

$$
\Delta E(L)=q^{a} \vec{v} \cdot \int_{-\infty}^{\infty} \frac{d \omega}{\omega} \int \frac{d^{3} \vec{k}}{4 \pi^{3} i} \int_{0}^{L / v} d t e^{-i K \cdot V t}\left[\frac{\vec{j}_{L}^{a}}{\epsilon_{L}}+\frac{\vec{j}_{T}^{a}}{\epsilon_{T}-k^{2} / \omega^{2}}\right]_{\text {ind }} .
$$

We stress that the latter expression is valid in the abelian approximation for the hard parton dynamics and within linear response theory, implying that $|\Delta E(L)|$ should be small compared to the initial parton energy $E$, which is also consistent with the assumption of a constant velocity.

If as in [3] the current (2.2) is used in (2.8), the exponential factor equals unity, and the collisional energy loss on the distance $L$ is uniquely determined by its rate per unit distance $(-d E / d x)_{\infty}=-\Delta E / L$. Inserting instead (2.5) in (2.8), and performing the time integral, we obtain the collisional energy loss $-\Delta E(L)$ of a hard parton produced at $t=0$ and travelling the distance $L$ in the medium,

$$
\frac{-\Delta E(L)}{C_{R} \alpha_{s}}=i \int_{-\infty}^{\infty} \frac{d \omega}{\omega} \int \frac{d^{3} \vec{k}}{4 \pi^{3}}\left[\frac{\vec{v}_{L}^{2}}{\epsilon_{L}}+\frac{\vec{v}_{T}^{2}}{\epsilon_{T}-k^{2} / \omega^{2}}\right]_{\text {ind }}\left\{\frac{1-e^{-i K . V L / v}}{K \cdot V(K . V+i \eta)}\right\} .
$$


We note that the factor between the curly brackets in (2.9) can be rewritten as

$$
\left\{\pi \delta(K . V) L / v+2 \frac{\sin ^{2}(K . V L /(2 v))}{(K . V)^{2}}+i \frac{\sin (K . V L / v)}{K . V} \mathrm{P}\left(\frac{1}{K . V}\right)\right\} .
$$

Using the following identities,

$$
\frac{\sin (u L)}{u} \underset{L \rightarrow \infty}{\longrightarrow} \pi \delta(u) \quad ; \quad \frac{\sin ^{2}(u L)}{L u^{2}} \underset{L \rightarrow \infty}{\longrightarrow} \pi \delta(u),
$$

we find that in the $L \rightarrow \infty$ limit, the expression (2.10) is equivalent to $2 \pi \delta(K . V) L / v$, and $(2.9)$ thus reproduces the result for $(-d E / d x)_{\infty}$ obtained in Ref. [3]. In the small $L$ limit, however, the second term of (2.10) is subleading and (2.10) reduces to $(i \mathrm{P}(1 / K . V)+$ $\pi \delta(K . V)) L / v$ as can be seen also directly from (2.9), thus leading to a modification of collisional energy loss at finite $L$.

\section{The retardation effect}

In this section we first express the energy loss (2.9) in terms of the discontinuity (on the real axis) of the longitudinal and transverse thermal gluon propagators. We then present and discuss our numerical results, which show that the asymptotic large $L$ behaviour of $-\Delta E(L)$ sets in only after some retardation time.

\subsection{Expression of $-\Delta E(L)$ in terms of thermal gluon spectral densities}

The dielectric functions can be expressed in terms of the longitudinal and transverse thermal gluon self-energies ${ }^{3}$,

$$
\epsilon_{L}=1+\Pi_{L}(x) / k^{2} \quad ; \quad \epsilon_{T}=1-\Pi_{T}(x) / \omega^{2},
$$

where $x=\omega / k$ and $\Pi_{L, T}$ have been obtained in the HTL approximation [14, 15],

$$
\Pi_{L}(x)=m_{D}^{2}\left[1-\frac{x}{2} \log \left(\frac{x+1}{x-1}\right)\right] \quad ; \quad \Pi_{T}(x)=\frac{1}{2} m_{D}^{2} x^{2}\left[1-\frac{x^{2}-1}{2 x} \log \left(\frac{x+1}{x-1}\right)\right] .
$$

The Debye mass denoted as $m_{D}$ is given by $m_{D}^{2}=4 \pi \alpha_{s} T^{2}\left(1+n_{f} / 6\right.$ ) (with $n_{f}=2$ the number of thermally equilibrated flavours). We also use the longitudinal and transverse thermal gluon propagators

$$
\Delta_{L}(\omega=k x, k)=\frac{-1}{k^{2}+\Pi_{L}(x)} \quad ; \quad \Delta_{T}(\omega=k x, k)=\frac{-1}{\omega^{2}-k^{2}-\Pi_{T}(x)} .
$$

Using (3.1) and (3.3) the expression (2.9) becomes (with $\vec{v}_{L}^{2}=v^{2} \cos ^{2} \theta, \vec{v}_{T}^{2}=v^{2} \sin ^{2} \theta$ ):

$$
\begin{aligned}
\frac{-\Delta E(L)}{C_{R} \alpha_{s}}=-i v^{2} \int \frac{d^{3} \vec{k}}{4 \pi^{3}} \int_{-\infty}^{\infty} \frac{d \omega}{\omega} & {\left[k^{2} \cos ^{2} \theta \Delta_{L}(\omega, k)+\omega^{2} \sin ^{2} \theta \Delta_{T}(\omega, k)\right]_{\text {ind }} } \\
\times & \left.\times \frac{1-e^{-i(\omega-k v \cos \theta) L / v}}{(\omega-k v \cos \theta)(\omega-k v \cos \theta+i \eta)}\right\} .
\end{aligned}
$$

\footnotetext{
${ }^{3}$ In the following, we will use the sign conventions and notations of Ref. 15.
} 
The longitudinal and transverse thermal gluon propagators have singularities on the real $\omega$-axis, namely branch cuts (corresponding to Landau damping) in the spacelike $|x|<1$ region, and poles corresponding to collective excitations of the plasma (plasmons) in the timelike $|x|>1$ region. Those singularities must be treated using the retarded prescription $\omega \rightarrow \omega+i \eta$ arising in the analytical continuation from imaginary to real frequencies in finite temperature field theory. As we explained in section 2.2, the potential $1 / \omega$ singularity at $\omega \rightarrow 0$ must be also regularized with the retarded prescription. Thus all singularities on the real axis appearing in (3.4) are implicitly written with the same, retarded prescription.

It is convenient to perform the $\omega$-integral in (3.4) using Cauchy's theorem, by closing the integration contour in the lower (complex $\omega$ ) half-plane, as required by the presence of the exponential factor in the integrand (since $L>0$ ). Thus the integral over the real axis is replaced by the integral over the (clockwise-going) contours around the singularities which lie just below the real axis, namely the poles at $\omega=-i \eta$ and $\omega=k v \cos \theta-i \eta$, and the plasmon poles and cuts of the propagators $\Delta_{L, T}$. We obtain from (3.4):

$$
\begin{aligned}
\frac{-\Delta E(L)}{C_{R} \alpha_{s}}= & -i v^{2} \int \frac{d^{3} \vec{k}}{4 \pi^{3}}\left\{(-2 i \pi) \frac{1}{v^{2}}\left(1-e^{i k L \cos \theta}\right) \operatorname{Re} \Delta_{L}(0, k)\right. \\
+ & +(-2 i \pi) \frac{i L k \cos \theta}{v^{2}}\left[\operatorname{Re} \Delta_{L}(k v \cos \theta, k)+v^{2} \sin ^{2} \theta \operatorname{Re} \Delta_{T}(k v \cos \theta, k)\right] \\
+ & 2 i \int_{-\infty}^{\infty} d \omega\left[k^{2} \cos ^{2} \theta \operatorname{Im} \Delta_{L}(\omega, k)+\omega^{2} \sin ^{2} \theta \operatorname{Im} \Delta_{T}(\omega, k)\right] \\
& \left.\times \mathrm{P}\left(\frac{1}{\omega}\right) \mathrm{P}\left(\frac{1}{\omega-k v \cos \theta}\right) \frac{1-e^{-i(\omega-k v \cos \theta) L / v}}{\omega-k v \cos \theta}\right\}_{\text {ind }} .
\end{aligned}
$$

Due to the fact that $\operatorname{Re} \Delta_{L, T}(\omega, k)$ is an even function of $\omega$, the second line of the latter equation vanishes after angular integration. Recalling [15] that the spectral densities (with $s=L$ or $T$ )

$$
\rho_{s}(\omega, k) \equiv 2 \operatorname{Im} \Delta_{s}(\omega+i \eta, k)=2 \pi \operatorname{sgn}(\omega) z_{s}(k) \delta\left(\omega^{2}-\omega_{s}^{2}(k)\right)+\beta_{s}(\omega, k) \theta\left(k^{2}-\omega^{2}\right)
$$

vanish at $\omega=0$, we can rewrite (3.5) in the form:

$$
\begin{aligned}
\frac{-\Delta E(L)}{C_{R} \alpha_{s}}=\int \frac{d^{3} \vec{k}}{2 \pi^{2}}\left\{\frac{1-\cos (k L \cos \theta)}{k^{2}+m_{D}^{2}}+\right. & v^{2} \int_{-\infty}^{\infty} \frac{d \omega}{2 \pi \omega}\left[k^{2} \cos ^{2} \theta \rho_{L}+\omega^{2} \sin ^{2} \theta \rho_{T}\right] \\
& \left.\times 2 \frac{\sin ^{2}((\omega-k v \cos \theta) L /(2 v))}{(\omega-k v \cos \theta)^{2}}\right\}_{\text {ind }} .
\end{aligned}
$$

The expression (3.7) for the collisional energy loss is actually ultraviolet divergent. The logarithmic UV divergence $\sim \int d k / k$ appears in the (leading) asymptotic $L \rightarrow \infty$ behaviour of (3.7),

$$
\frac{-\Delta E_{\infty}(L)}{C_{R} \alpha_{s}}=v^{2} \int \frac{d^{3} \vec{k}}{2 \pi^{2}} \int_{-\infty}^{\infty} \frac{d \omega}{2 \pi \omega}\left[k^{2} \cos ^{2} \theta \rho_{L}+\omega^{2} \sin ^{2} \theta \rho_{T}\right]_{\text {ind }}\left[\frac{\pi L}{v} \delta(\omega-k v \cos \theta)\right] .
$$


In Ref. 酒 it is stressed that the macroscopic description is meaningful only for distant collisions, and a framework which properly includes close collisions is formulated. Within such an approach the energy loss is UV convergent, but receives a contribution from the 'hard' domain $k \gg T$. In QCD the running of $\alpha_{s}$ improves the UV convergence, and the stationary rate of energy loss $(-d E / d x)_{\infty}=-\Delta E_{\infty}(L) / L$ is expected to be actually dominated by the 'soft' $k \sim m_{D}$ region [5]. We will come back to this point in the end of section 3.2. In the absence of a rigorous treatment with running coupling, we choose to focus on the difference between $-\Delta E(L)$ and the standard stationary law (3.8),

$$
\begin{aligned}
d(L) & =-\Delta E(L)+\Delta E_{\infty}(L) \\
\frac{d(L)}{C_{R} \alpha_{s}}=\int \frac{d^{3} \vec{k}}{2 \pi^{2}}\left\{\frac{1-}{\cos (k L \cos \theta)}\right. & k^{2}+m_{D}^{2} \\
\quad & {\left.\left[2 \frac{\sin ^{2}((\omega-k v \cos \theta) L /(2 v))}{(\omega-k v \cos \theta)^{2}}-\frac{d \omega}{v} \delta(\omega-k v \cos \theta)\right]\right\}_{\text {ind }} }
\end{aligned}
$$

which turns out to be UV convergent (see Appendix A). As discussed in the next section, we also expect the main feature of the function $d(L)$ (which we evaluate with fixed coupling), namely its limiting value when $L \rightarrow \infty$, to be unaffected by the running of $\alpha_{s}$.

\subsection{Numerical results and discussion}

In this section we first discuss the main features of $d(L)$, in particular its large $L$ limit, and the domain of validity of our calculation. (The mathematical properties of $d(L)$ are studied in the Appendices.) Consequences on the phenomenology of the collisional loss $-\Delta E(L)$ are then presented.

The large $L$ limit of $d(L)$,

$$
d_{\infty} \equiv \lim _{L \rightarrow \infty} d(L)=\lim _{L \rightarrow \infty}\left[-\Delta E(L)+\Delta E_{\infty}(L)\right],
$$

is calculated exactly in Appendix B (see Eqs. (B.13), (B.15) and $(\overline{B .17})$ ) and can be accurately approximated by

$$
d_{\infty} \approx-C_{R} \alpha_{s} m_{D}(1+\sqrt{2}(\gamma-1))
$$

Our central result is that $d_{\infty}$ scales as $\gamma=1 / \sqrt{1-v^{2}}$ when $v=p / E \rightarrow 1$. The largeness (and negative sign) of $d_{\infty}$ for large quark energies will be the main reason for the important delay of the stationary regime. The $L$-dependence of $d(L)$ is presented in Fig. I for a fast charm quark. The function $d(L)$ is found to be negative for all $L$, and Fig. 孟 shows the increase (in magnitude) of $d_{\infty}$ with the quark energy, as found analytically.

The observed values of $d_{\infty}$ can be translated to an estimate of the time scale characteristic of the transitory regime by defining the retardation time

$$
t_{\mathrm{ret}}=d_{\infty} /(d E / d x)_{\infty}
$$

For a quark with $p=10 \mathrm{GeV},-d_{\infty} \sim 1 \mathrm{GeV}$ and $-(d E / d x)_{\infty} \sim 0.1-0.2 \mathrm{GeV} / \mathrm{fm}$ yields $t_{\text {ret }} \sim 5-10 \mathrm{fm}$. This number should be considered as a rough estimate, due to the 


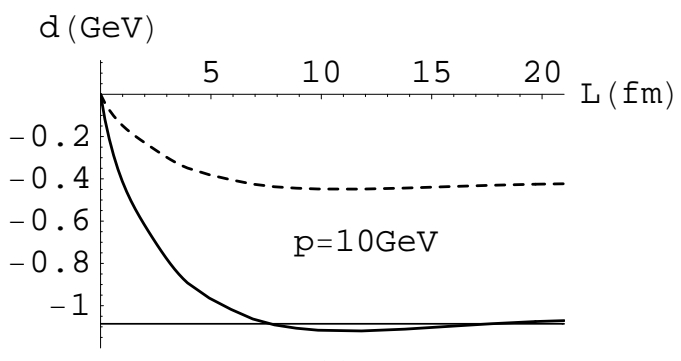

(a)

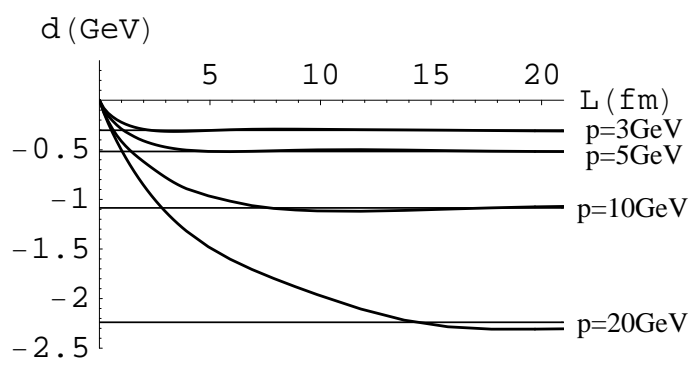

(b)

Figure 1: The function $d(L)$ defined by (3.9) and (3.10) for a charm quark $(M=1.5 \mathrm{GeV})$ produced in a QGP of temperature $T=0.25 \mathrm{GeV}$, as a function of the distance $L$ travelled in the plasma. We use $\alpha_{s}=0.2$ and $n_{f}=2$ in the expression of the Debye mass $m_{D}^{2}=4 \pi \alpha_{s} T^{2}\left(1+n_{f} / 6\right)$, giving $m_{D} \simeq 0.46 \mathrm{GeV}$. (a) $d(L)$ for a quark momentum $p=10 \mathrm{GeV}$ (full line). The contribution from initial bremsstrahlung (see section 4 and Appendix B) to $d(L)$ is represented by the dashed line. (b) Dependence of $d(L)$ on the charm quark momentum $p$. The thin straight lines give the values of $d_{\infty}$ for the corresponding values of $p$.

numerous approximations used in our theoretical model. But we stress that the retardation time may be of the order of several fm for $p=10 \mathrm{GeV}$, and that it scales with the quark energy for large energies.

The scaling in $\gamma$ of $d_{\infty}$ (when $\gamma \rightarrow \infty$ ) results in a similar scaling of the retardation time, $t_{\text {ret }} \sim \gamma / m_{D}$, and this has the following simple interpretation. The stationary regime for energy loss sets in when the regions of the plasma at a transverse distance $\sim 1 / m_{D}$, polarized by the parton current, start to retroact on the parton. When the parton velocity $v$ is small, this takes a time $t_{\text {ret }} \sim 1 / m_{D}$. When $v \rightarrow 1$, the latter scenario can occur only if the parton is not ahead of the relevant polarized regions after the time $t_{\text {ret }}$. At initial time $t=0$, this requires the parton to polarize a domain which is ahead of it, at an angle $\theta \sim \sqrt{1-v^{2}}=1 / \gamma \ll 1$ with respect to the direction of $\vec{v}$. The time $t_{\text {ret }}$ corresponds to the time necessary to send a signal at a transverse distance $\sim 1 / m_{D}$ in the direction $\theta$, leading to $t_{\text {ret }} \sim 1 /\left(m_{D} \theta\right)$ for $\theta \ll 1$, hence $t_{\text {ret }} \sim \gamma / m_{D}$ when $v \rightarrow 1$.

We now discuss the domain of validity of our calculation. As shown in Appendix B (see $(\mathrm{B} .18)$ ), the typical values of $k$ contributing to $d_{\infty}$ are $k \sim \mathcal{O}\left(\gamma m_{D}\right)$. Since using the HTL gluon spectral densities in (3.10) a priori requires $k \ll T$ [14, 15], our calculation of $d_{\infty}$ would seem to be justified, in the perturbative framework $m_{D} \propto g T \ll T$, only provided $\gamma$ is not too large, $\gamma \ll 1 / g$. However, as can be easily inferred from Appendix $\mathrm{B}$, the dominant contribution to $d_{\infty}$ arises from $\left|\omega^{2}-k^{2}\right| \sim m_{D}^{2}$ (with $\omega \simeq k \sim \gamma m_{D}$ ), i.e. from the region where $K=(\omega, k)$ is close to its mass-shell. It is known [19, 20] that in this region of low virtualities the HTL gluon propagator is a very good approximation to the exact propagator even in the domain $k \gg T$. Thus our calculation of $d_{\infty}$ might be justified for all values of $\gamma$.

Contrary to the position of the asymptote $d_{\infty}$, the small $L$ behaviour of $d(L)$ seen on Fig. 1 should not be physically sound. The small $L$ behaviour of $d(L)$ can be obtained 

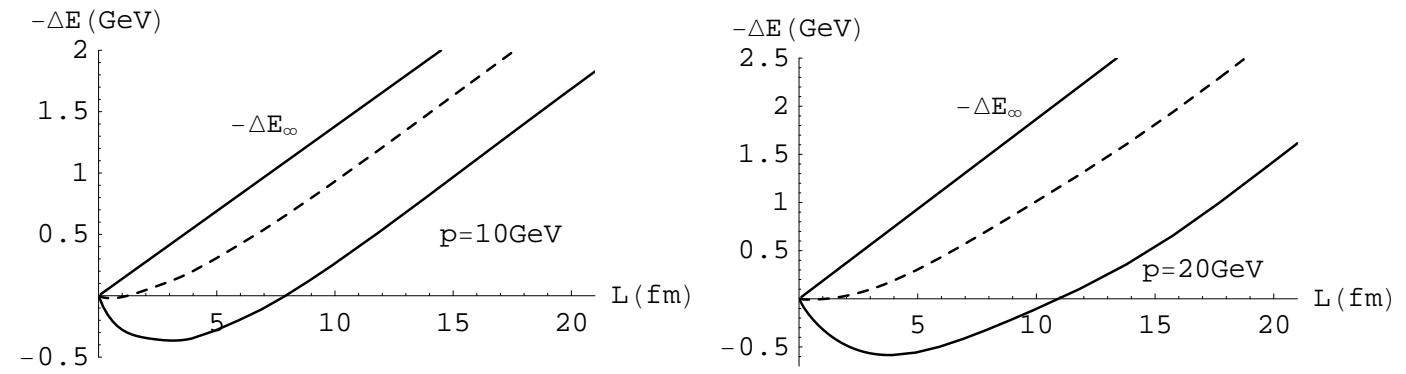

Figure 2: Collisional energy loss of a charm quark produced in a QGP as a function of the distance $L$ travelled in the plasma, for $p=10 \mathrm{GeV}$ and $p=20 \mathrm{GeV}$. The total result (full line) is compared to the linear law $\left(-\Delta E_{\infty}\right)$ corresponding to (3.14), and which would arise in Ref. 唒 by evaluating the slope to logarithmic accuracy. As in Fig. 1 la the dashed line singles out the contribution to $-\Delta E(L)$ from initial bremsstrahlung (see section 4).

for instance from (B.4), and is of the form $d\left(L \ll m_{D}^{-1}\right) \propto \alpha_{s}^{2} T^{2} L \log \left(m_{D} L\right)$, where the logarithm arises from an integral $\sim \int_{m_{D}}^{1 / L} d k / k$. Thus the small $L$ limit is sensitive to large virtualities $\left|\omega^{2}-k^{2}\right| \sim k^{2} \gg T^{2}$, where the HTL approximation becomes invalid.

In order to qualitatively illustrate how $d(L)$ delays the collisional energy loss, we have to add the stationary contribution $-\Delta E_{\infty}(L)$ given in (3.8). In the end of section 3.1 we pointed out that (3.8) is ill-defined as it stands, because of a logarithmic UV divergence. We mentioned that this divergence would be absent, either with a proper treatment of close collisions with fixed $\alpha_{s}$ [ [hardness of $k$ contributing to $-(d E / d x)_{\infty}$. This stresses the need for a rigorous treatment with running $\alpha_{s}$. In the absence of such a treatment, and since we need the stationary loss $-\Delta E_{\infty}(L)$ only for a qualitative illustration of the retardation effect, we will use the 'standard' result derived in Ref. [4], which reads to logarithmic accuracy (which is sufficient for our purpose):

$$
\begin{gathered}
\left(-\frac{d E}{d x}\right)_{\infty} \equiv \frac{-\Delta E_{\infty}(L)}{L}=\frac{C_{R} \alpha_{s} m_{D}^{2}}{2}\left[\frac{1}{v}-\frac{1-v^{2}}{2 v^{2}} \log \left(\frac{1+v}{1-v}\right)\right] \log \left(\frac{k_{\max }}{m_{g}}\right), \\
\text { where } k_{\max } \equiv \operatorname{Min}\left\{\frac{E T}{M}, \sqrt{E T}\right\},
\end{gathered}
$$

and $m_{g}=m_{D} / \sqrt{3}$ is the gluon thermal mass.

Our results for $-\Delta E(L)$ given by (3.9), (3.10), (3.14) and (3.15) are shown in Fig. 2 , illustrating the main qualitative feature, i.e. the important delay of the stationary regime. The retardation time (3.13), which corresponds to the value of $L$ where the asymptote of $-\Delta E(L)$ cuts the horizontal axis, is close to the intersection of the curve $-\Delta E(L)$ with this axis.

As another consequence of the large negative values of $d(L)$, we observe on Fig. 2 that the induced energy loss $-\Delta E(L)$ is negative at relatively small $L$, and thus corresponds to an (induced) energy gain. 
Two effects may explain the latter observation, which goes beyond our initial expectation of a delayed but however positive energy loss. First, we recall that current conservation requires the energetic parton to be produced with at least another parton. In the simple case of a color singlet dipole we considered (see (2.5)), we expect the dipole to separate more easily in the medium than in vacuum, due to charge screening. Secondly, the current created at $t=0$ produces radiation ${ }^{4}$. As discussed in detail in section 4 , in the medium the radiated energy corresponds to the excitation of the QGP plasmon modes and is reduced compared to vacuum (the induced radiated energy is negative), as might have been expected for massive modes. However, the retardation effect is only partly due to this difference between in-medium and vacuum radiation, as seen in Fig. 17a and further discussed in section 4.

We end this section by discussing which features of our calculation might be affected by the running of $\alpha_{s}$.

First, the standard result [4] for $-\Delta E_{\infty}(L)$ we have used arises from the logarithmic interval $m_{g} \ll k \ll k_{\max }$ (see (3.14) and (3.15)). In QCD, with a running coupling evaluated at a scale on the order of the gluon virtuality, we expect $(-d E / d x)_{\infty}$ to be $\propto \int d k \alpha_{s}(k)^{2} / k \propto \int d k /\left(k \log ^{2} k\right)$, showing that $(-d E / d x)_{\infty}$ depends negligibly on $E$ when $E \rightarrow \infty$ [5]. (In this sense the $\log k_{\max } \propto \log E$ factor in (3.14) is an artefact of the fixed coupling approximation.) Most importantly, $(-d E / d x)_{\infty}$ is actually dominated by the soft (infrared) region $k \sim m_{D}$ when $E \gg m_{D}$ [5], and we expect the calculation of $(-d E / d x)_{\infty}$ within a macroscopic description and with running $\alpha_{s}$ to be self-consistent.

Secondly, the running of $\alpha_{s}$ should affect the behaviour of $d(L)$ at small $L<1 / m_{D}$. We discussed previously that since the slope of $d(L)$ at small $L$ is of the form $\sim \alpha_{s}^{2} \int_{m_{D}}^{1 / L} d k / k$, it cannot be consistently derived within a macroscopic description. This should not be the case with running coupling, since we expect the slope to be rather $\sim \int_{m_{D}}^{1 / L} d k /\left(k \log ^{2} k\right)$, which is dominated by $k \sim m_{D}$ and independent of $L$ when $L \rightarrow 0$. Thus the sharp behaviour $d(L \rightarrow 0) \propto L \log L$ obtained for fixed $\alpha_{s}$ should become $d(L \rightarrow 0) \propto-\alpha_{s}\left(m_{D}\right) T^{2} L$ in the case of running $\alpha_{s}$. As a consequence, the energy loss $-\Delta E(L)=-\Delta E_{\infty}(L)+d(L)$ shown in Fig. 2 should be at most $^{5} \sim \mathcal{O}(L)$ at small $L$. Thus, the relatively important induced energy gain at small $L$ seen in Fig. 2 might be strongly affected by the running of the coupling. In contradistinction, we stress that the large $L$ limit $d_{\infty}$ of $d(L)$ should not be affected by the running of $\alpha_{s}$, since $d_{\infty}$ depends on low virtualities $\left|\omega^{2}-k^{2}\right| \sim m_{D}^{2}$.

\section{Induced radiation}

As already mentioned, and as is well-known, the sudden acceleration of the energetic parton at time $t=0$ comes along with bremsstrahlung radiation. The quantity $-\Delta E(L)$ which we called induced collisional energy loss actually contains this contribution. In this section

\footnotetext{
${ }^{4}$ The induced radiated energy is part of $-\Delta E(L)$ as we defined it (see section 4 ).

${ }^{5}$ In fact, for running coupling the slope of $-\Delta E(L)$ is expected to vanish when $L \rightarrow 0$. This can be seen by expanding the bracket in (2.9) to order $L$. The UV convergence of the integral over $k$ being ensured by the running of the coupling, the integral over $\omega$ can be performed by closing the contour in the upper half-plane where there is no singularity and identically vanishes.
} 

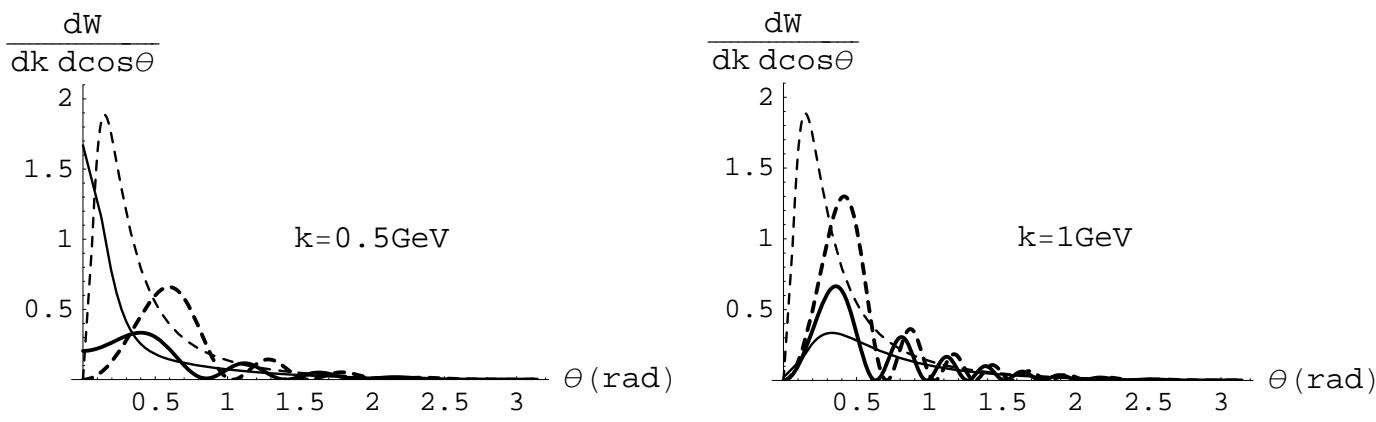

Figure 3: In-medium angular radiation spectrum (broad full line) (1.2) for $L=5 \mathrm{fm}, p=10 \mathrm{GeV}$, $k=0.5 \mathrm{GeV}$ (left) and $k=1 \mathrm{GeV}$ (right). The thin full line represents the in-medium spectrum in the $L \rightarrow \infty$ limit (4.3). The broad and thin dashed lines represent the same spectra in the vacuum, given respectively by 4.4 and $(4.5)$.

we single out this radiative component from $-\Delta E(L)$ and emphasize that it plays only a finite part in the retardation effect studied in section 3 .

In order to obtain the contribution $W(L)$ to $-\Delta E(L)$ originating from radiation, we single out in (3.4) the contribution of the plasmon poles. This is done by using the expression of the gluon propagators close to their poles [15], i.e. at $\omega^{2} \simeq \omega_{s}^{2}(k)$,

$$
\Delta_{s}(\omega, k) \simeq \frac{-z_{s}(k)}{(\omega+i \eta)^{2}-\omega_{s}^{2}(k)}=-z_{s}(k)\left[\mathrm{P}\left(\frac{1}{\omega^{2}-\omega_{s}^{2}(k)}\right)-i \pi \operatorname{sgn}(\omega) \delta\left(\omega^{2}-\omega_{s}^{2}(k)\right)\right],
$$

and picking only the $\delta$-term in the latter. From (3.4) we obtain the total (i.e., we do not subtract the vacuum contribution from it) in-medium radiation spectrum:

$$
\begin{gathered}
\frac{d W(L)}{d k d \cos \theta}=\frac{C_{R} \alpha_{s}}{\pi}\left\{z_{L}(k) \frac{k^{2}}{\omega_{L}^{2}(k)} \cos ^{2} \theta \frac{\sin ^{2}\left(\left(\omega_{L}(k)-k v \cos \theta\right) L /(2 v)\right)}{\left(\cos \theta-\omega_{L}(k) /(k v)\right)^{2}}\right. \\
\left.\quad+z_{T}(k) \sin ^{2} \theta \frac{\sin ^{2}\left(\left(\omega_{T}(k)-k v \cos \theta\right) L /(2 v)\right)}{\left(\cos \theta-\omega_{T}(k) /(k v)\right)^{2}}\right\} \\
\underset{L \rightarrow \infty}{\longrightarrow} \frac{C_{R} \alpha_{s}}{2 \pi}\left\{z_{L}(k) \frac{k^{2}}{\omega_{L}^{2}(k)} \frac{\sin ^{2} \theta}{\left(\cos \theta-\omega_{L}(k) /(k v)\right)^{2}}+z_{T}(k) \frac{\left.\cos _{T}(k) /(k v)\right)^{2}}{\left(\cos \theta-\omega_{T}\right.}\right\}
\end{gathered}
$$

where the functions $\omega_{L, T}(k)$ and $z_{L, T}(k)$ can be found in Ref. [15].

The differential spectrum in the polar angle $\theta$ is represented in Fig. 3 for $L=5 \mathrm{fm}$, $p=10 \mathrm{GeV}$, and for two values of $k, k=0.5 \mathrm{GeV}$ and $k=1 \mathrm{GeV}$. The vacuum contribution is obtained by setting $z_{L}=0, z_{T}=1, \omega_{L}(k)=\omega_{T}(k)=k$ in (4.2), yielding:

$$
\begin{aligned}
\left.\frac{d W(L)}{d k d \cos \theta}\right|_{v a c} & =\frac{C_{R} \alpha_{s}}{\pi} \sin ^{2} \theta \frac{\sin ^{2}((k-k v \cos \theta) L /(2 v))}{(\cos \theta-1 / v)^{2}} \\
& \underset{L \rightarrow \infty}{\longrightarrow} \frac{C_{R} \alpha_{s}}{2 \pi} \frac{v^{2} \sin ^{2} \theta}{(1-v \cos \theta)^{2}} .
\end{aligned}
$$

As expected, (4.5) corresponds to the vacuum bremsstrahlung spectrum of a single charge suddenly accelerated at $t=0$. We remark that the latter spectrum contributes to the 
radiative energy loss induced by parton multiple scattering [6, 7, 8], as the zeroth order in the number of parton rescatterings ('self-quenching' [8]). As already mentioned, the corresponding in-medium radiation spectrum (4.3) arises from medium polarization, and thus differs from the spectrum induced by (at least one) rescattering calculated in [6, 0, 8]. The (large $L$ ) in-medium radiation spectrum (4.3) was previously obtained in Ref. 21] within a diagrammatic approach.

In vacuum the spectrum (4.5) is modified by parton cascade and hadronization, but the angular pattern (4.5) in principle affects the distribution of final state hadrons. In a QGP of very large size, this distribution will rather be sensitive to the large $L$ in-medium spectrum (4.3). Similarly, the modification of the finite $L$ diffraction pattern when one goes from vacuum (spectrum (4.4)) to the medium (spectrum (4.2)) may be of some relevance to discuss particle production induced by a fast parton travelling a distance $L$ in the QGP. When $k$ is large, the spectrum (4.2) approaches the vacuum spectrum (4.4). This can already be seen for $k=1 \mathrm{GeV}$ (Fig. 3 right), although some attenuation is still visible at small $\theta$. When $k$ decreases (Fig. 3 left), a strong distorsion shows up as (4.2) becomes sensitive to the plasmon modes of the QGP.

However the spectrum (4.2) is not directly observable (not speaking of parton cascade nor hadronization). In a realistic situation, the parton propagates in the thermal medium for $0 \leq t \leq L / v$, and in vacuum for $t \geq L / v$. Thus the full picture should incorporate both stages. In addition, the fast parton produces transition radiation when going through the discontinuity between the thermal medium $\left(\epsilon_{L, T} \neq 1\right)$ and the vacuum $\left(\epsilon_{L, T}=1\right)$. This transition radiation must also be taken into account in order to obtain the complete radiation spectrum. We postpone to a future work the study of transition radiation in our context, as well as its influence on the overall energy loss. In particular, it is too early to possibly relate the angular spectrum (4.2) (see Fig. 3) to the humpbacked azimuthal distribution of particles produced back to the leading jet observed at RHIC [22]. However, the angular radiation spectrum (4.2) shown in Fig. 3 suggests that (at least some component of) the particle yield in heavy ion collisions should exhibit a diffraction pattern arising from the finite length of the medium along which collisional energy loss occurs.

Finally, we stress that in our calculation the radiated energy (obtained by integrating (4.2) over the radiated gluon energy and emission angle) is smaller in the medium than in vacuum. This feature could be foreseen by comparing the in-medium and vacuum differential spectra in Fig. 3. Thus the contribution of induced radiation to what we defined as the induced collisional energy loss $-\Delta E(L)$ is negative. Numerically, we find that the bremsstrahlung contribution always accounts for less than $50 \%$ of the difference $d(L)$ between $-\Delta E(L)$ and the asymptotic result $-\Delta E_{\infty}$ (see Figs. 17a and 2). In the end of Appendix B we show that the relative contribution from bremsstrahlung to $d(L)$ reaches exactly $1 / 2$ when $L \rightarrow \infty$ and in the ultrarelativistic limit $v \rightarrow 1$. This shows that the retardation effect studied in section 3 cannot be solely attributed to the radiative component of $-\Delta E(L)$. 


\section{Conclusion}

We have studied the collisional energy loss $-\Delta E(L)$, in the fixed coupling approximation, of an energetic parton travelling the distance $L$ in a QGP, and initially produced (at $t=0)$ in the medium. Compared to previous estimates which assumed the parton to be produced at $t=-\infty,-\Delta E(L)$ is strongly suppressed up to $L \sim t_{\text {ret }}$, where the retardation time $t_{\text {ret }}$ scales with the parton momentum $p$ at large $p$. For $p=10 \mathrm{GeV}$ we roughly estimated $t_{\text {ret }} \sim 5-10 \mathrm{fm}$. We stress that this estimate is only indicative due to the various approximations used in our theoretical model, in particular the small coupling limit $g \ll 1$. Also, the running of $\alpha_{s}$ is expected to affect both the small $L$ behaviour of $-\Delta E(L)$, and the asymptotic stationary rate $(-d E / d x)_{\infty}$, as discussed in the end of section 3.2. A rigorous treatment with running $\alpha_{s}$ would be needed to obtain a better quantitative estimate of $t_{\text {ret }}$.

We believe our results could be relevant to jet quenching phenomenology, since it has recently been argued [9, 11] that collisional energy loss should be reconsidered as an important source of energy loss. In addition to the suppression of $-\Delta E(L)$, we find that the asymptotic linear behaviour of $-\Delta E(L)$ is delayed to about $L \sim t_{\text {ret }}$. In particular, using a stationary energy loss rate $-d E / d x$ makes sense only for quite large values of $L>t_{\text {ret }}$.

The suppression and retardation of $-\Delta E(L)$ are encoded in the difference $d(L)=$ $-\Delta E(L)+\Delta E_{\infty}(L)$ between $-\Delta E(L)$ and the 'standard' stationary result $-\Delta E_{\infty}(L) \propto L$. As we have shown, $d(L)$ is a well-defined (UV convergent) quantity, and our main result the largeness of the retardation time - is independent of the uncertainties on $(-d E / d x)_{\infty}$ and on the precise shape of $-\Delta E(L)$ at small $L$. The main reason for the large magnitude of the retardation time is the scaling $d_{\infty} \propto-\gamma m_{D}$ when $\gamma=E / M \rightarrow \infty$, where $d_{\infty}$ is the large $L$ limit of $d(L)$. We showed that the (induced) bremsstrahlung arising from the hard parton being suddenly accelerated at $t=0$, formally included in $d(L)$, contributes only partly to $d_{\infty}$. Thus the retardation effect cannot be solely attributed to initial radiation. The scaling in $\gamma$ of $d_{\infty}$ (when $\gamma \rightarrow \infty$ ) results in a similar scaling of the retardation time, $t_{\text {ret }} \sim \gamma / m_{D}$, and a physical interpretation of this fact is given in section 3.2.

Finally, we mention that in order to explain the observed dependence [23] in azimuthal angle $\phi$ (with respect to the reaction plane in a heavy ion $A A$ collision) of the nuclear modification factor $R_{A A}$, taking into account the geometry of the collision is not sufficient [24. A length scale $L \simeq 2 \mathrm{fm}$ has to be introduced, below which the parent parton of the high $p_{T}$ jet or hadron is assumed to be insensitive to energy loss. In Ref. 24] this parameter is interpreted as the formation time of the plasma, but it is also stressed there that $2 \mathrm{fm}$ is quite large compared to the values $\sim 0.2 \mathrm{fm}$ usually taken for the plasma formation time. Our study suggests that this parameter might instead hint to the possibility of a negative loss $-\Delta E_{\text {coll }}(L)$ before the stationary regime, partially compensating the radiative energy loss $-\Delta E_{\text {rad }}(L)$ induced by rescattering of the energetic parton. 


\section{Acknowledgments}

We would like to warmly thank D. Schiff, Y. L. Dokshitzer and A. Peshier for very instruc-

tive and helpful discussions. We are also grateful to J. Aichelin for stimulating exchanges during this work.

\section{APPENDIX}

\section{A. Ultraviolet convergence of the function $d(L)$}

Here we show that $d(L)$ given by $(3.10)$ is ultraviolet convergent. For this purpose, it is sufficient to prove that the angular integral

$$
I=\int_{-1}^{1} d \cos \theta f(\cos \theta)\left[2 \frac{\sin ^{2}\left(\frac{k L}{2 v}(x-v \cos \theta)\right)}{k^{2}(x-v \cos \theta)^{2}}-\frac{\pi L}{k v^{2}} \delta\left(\frac{x}{v}-\cos \theta\right)\right]
$$

where $x=\omega / k$ and $f(\cos \theta)=\cos ^{2} \theta$ or $\sin ^{2} \theta$, is of order $\sim \mathcal{O}\left(1 / k^{2}\right)$ when $k \rightarrow \infty$. Indeed, the second term of (A.1) is of order $\sim \mathcal{O}(1 / k)$, leading to the logarithmic UV divergence of $-\Delta E_{\infty}(L)$ (see (3.8)). With the change of variable

$$
u=\alpha(x-v \cos \theta) \quad ; \quad \alpha \equiv \frac{k L}{2 v}
$$

we obtain

$$
I=\frac{L}{k v^{2}}\left\{\int_{\alpha(x-v)}^{\alpha(x+v)} d u \frac{\sin ^{2} u}{u^{2}} f\left(\frac{x}{v}-\frac{u}{\alpha v}\right)-\pi \theta(v-|x|) f\left(\frac{x}{v}\right)\right\} \equiv I_{1}+I_{2},
$$

where we define

$$
\begin{aligned}
& I_{1}=\frac{L}{k v^{2}} f\left(\frac{x}{v}\right)\left[\int_{\alpha(x-v)}^{\alpha(x+v)} d u \frac{\sin ^{2} u}{u^{2}}-\pi \theta(v-|x|)\right], \\
& I_{2}=\frac{L}{k v^{2}} \int_{\alpha(x-v)}^{\alpha(x+v)} d u \frac{\sin ^{2} u}{u^{2}}\left(f\left(\frac{x}{v}-\frac{u}{\alpha v}\right)-f\left(\frac{x}{v}\right)\right) .
\end{aligned}
$$

The behaviour of $I_{1}$ when $\alpha=k L /(2 v) \rightarrow \infty$ is easily found by treating separately the two cases $|x|>v$ and $|x|<v$. In the first case we can replace $\sin ^{2} u \rightarrow 1 / 2$ in the integrand and we find

$$
I_{1} \underset{\alpha \rightarrow \infty}{\sim} \frac{L}{k v^{2}} f\left(\frac{x}{v}\right) \frac{v}{\alpha} \frac{1}{x^{2}-v^{2}}=\frac{2}{k^{2}} f\left(\frac{x}{v}\right) \frac{1}{x^{2}-v^{2}},
$$

which is $\sim \mathcal{O}\left(1 / k^{2}\right)$. In the second case, $|x|<v$, we write

$$
\int_{\alpha(x-v)}^{\alpha(x+v)} d u \frac{\sin ^{2} u}{u^{2}}-\pi=-\int_{\alpha(x+v)}^{\infty} d u \frac{\sin ^{2} u}{u^{2}}-\int_{-\infty}^{\alpha(x-v)} d u \frac{\sin ^{2} u}{u^{2}}
$$


where the replacement $\sin ^{2} u \rightarrow 1 / 2$ can be made in the r.h.s. when $\alpha \rightarrow \infty$, leading again to (A.6). Note that when $\alpha \rightarrow \infty$, the expression (A.4) is thus equivalent to

$$
I_{1} \underset{\alpha \rightarrow \infty}{\sim} \frac{L}{2 k v^{2}} f\left(\frac{x}{v}\right) \int_{\alpha(x-v)}^{\alpha(x+v)} d u \mathrm{P}\left(\frac{1}{u^{2}}\right) .
$$

In the $I_{2}$ integral defined in (A.5), the contribution from $u \ll \alpha$ is negligible, showing that typically $u \sim \alpha$. Thus we can replace $\sin ^{2} u \rightarrow 1 / 2$, yielding:

$$
I_{2} \underset{\alpha \rightarrow \infty}{\sim} \frac{L}{2 k v^{2}} \int_{\alpha(x-v)}^{\alpha(x+v)} d u \mathrm{P}\left(\frac{1}{u^{2}}\right)\left(f\left(\frac{x}{v}-\frac{u}{\alpha v}\right)-f\left(\frac{x}{v}\right)\right) .
$$

We infer from the above that the behaviour of (A.1) when $\alpha=k L /(2 v) \rightarrow \infty$ is obtained by the following replacement:

$$
\left[2 \frac{\sin ^{2}\left(\frac{k L}{2 v}(x-v \cos \theta)\right)}{k^{2}(x-v \cos \theta)^{2}}-\frac{\pi L}{k v^{2}} \delta\left(\frac{x}{v}-\cos \theta\right)\right] \underset{\alpha \rightarrow \infty}{\longrightarrow} \mathrm{P}\left(\frac{1}{k^{2}(x-v \cos \theta)^{2}}\right) .
$$

The r.h.s. is $\sim \mathcal{O}\left(1 / k^{2}\right)$, to be compared to $\mathcal{O}(L / k)$ (second term of (A.1)). This completes our proof of the UV convergence of the function $d(L)$.

\section{B. Large $L$ limit of $d(L)$}

Here we evaluate the limiting value of $d(L)$ (defined by (3.9)) when $L \rightarrow \infty$,

$$
d_{\infty} \equiv \lim _{L \rightarrow \infty} d(L)=\lim _{L \rightarrow \infty}\left[-\Delta E(L)+\Delta E_{\infty}(L)\right] .
$$

\section{B.1 Total result for $d_{\infty}$}

Using (3.4) (with the bracket expressed as in (2.10)) we obtain:

$$
\begin{aligned}
\frac{d(L)}{C_{R} \alpha_{s}} & =i v^{2} \int \frac{d^{3} \vec{k}}{4 \pi^{3}} \int_{-\infty}^{\infty} \frac{d \omega}{\omega}\left[k^{2} \cos ^{2} \theta \Delta_{L}(\omega, k)+\omega^{2} \sin ^{2} \theta \Delta_{T}(\omega, k)\right]_{\text {ind }} \\
& \times\left\{\pi \delta(K . V) L / v-2 \frac{\sin ^{2}(K . V L /(2 v))}{(K . V)^{2}}-i \frac{\sin (K . V L / v)}{K . V} \mathrm{P}\left(\frac{1}{K . V}\right)\right\} .
\end{aligned}
$$

We now use the fact that if we replace $L$ by $-L$ in (3.4), the integral over $\omega$, performed by closing the integration contour in the upper half-plane, vanishes identically because the singularities in $\omega$ all lie below the real axis:

$$
\begin{aligned}
0 & =i v^{2} \int \frac{d^{3} \vec{k}}{4 \pi^{3}} \int_{-\infty}^{\infty} \frac{d \omega}{\omega}\left[k^{2} \cos ^{2} \theta \Delta_{L}(\omega, k)+\omega^{2} \sin ^{2} \theta \Delta_{T}(\omega, k)\right]_{\mathrm{ind}} \\
& \times\left\{-\pi \delta(K . V) L / v+2 \frac{\sin ^{2}(K . V L /(2 v))}{(K . V)^{2}}-i \frac{\sin (K . V L / v)}{K . V} \mathrm{P}\left(\frac{1}{K . V}\right)\right\} .
\end{aligned}
$$

Adding (B.3) to (B.2) we get:

$$
\begin{aligned}
\frac{d(L)}{C_{R} \alpha_{s}}=i v^{2} \int \frac{d^{3} \vec{k}}{4 \pi^{3}} \int_{-\infty}^{\infty} \frac{d \omega}{\omega} & {\left[k^{2} \cos ^{2} \theta \Delta_{L}(\omega, k)+\omega^{2} \sin ^{2} \theta \Delta_{T}(\omega, k)\right]_{\text {ind }} } \\
\times & \left\{-2 i \frac{\sin (K \cdot V L / v)}{K . V} \mathrm{P}\left(\frac{1}{K . V}\right)\right\} .
\end{aligned}
$$


Using the first of the identities (2.11), and then $\delta(x) \mathrm{P}(1 / x)=-\delta^{\prime}(x)$, we obtain in the $L \rightarrow \infty$ limit:

$$
\frac{d_{\infty}}{C_{R} \alpha_{s}}=-\pi v^{2} \operatorname{Re} \int \frac{d^{3} \vec{k}}{2 \pi^{3}} \int_{-\infty}^{\infty} \frac{d \omega}{\omega}\left[k^{2} \cos ^{2} \theta \Delta_{L}(\omega, k)+\omega^{2} \sin ^{2} \theta \Delta_{T}(\omega, k)\right]_{\text {ind }} \delta^{\prime}(K . V) .
$$

In $\delta^{\prime}(K . V)$ we trade the derivative $\partial / \partial \omega$ for $\partial / \partial v$ :

$$
\frac{d_{\infty}}{C_{R} \alpha_{s}}=\pi v^{2} \frac{\partial}{\partial v} \frac{1}{v} \operatorname{Re} \int \frac{d^{3} \vec{k}}{2 \pi^{3}}\left[\Delta_{L}(k v \cos \theta, k)+v^{2} \sin ^{2} \theta \Delta_{T}(k v \cos \theta, k)\right]_{\mathrm{ind}} .
$$

Using (3.3) we have

$$
\begin{aligned}
{\left[\Delta_{L}(k x, k)\right]_{\text {ind }} } & =\frac{\Pi_{L}(x)}{k^{2}\left[k^{2}+\Pi_{L}(x)\right]} \\
{\left[\Delta_{T}(k x, k)\right]_{\text {ind }} } & =\frac{-\Pi_{T}(x)}{k^{2}\left(x^{2}-1\right)\left[k^{2}\left(x^{2}-1\right)-\Pi_{T}(x)\right]},
\end{aligned}
$$

and denoting $x=v \cos \theta$ the equation $(\overline{\mathrm{B} .6})$ becomes:

$$
\frac{d_{\infty}}{C_{R} \alpha_{s}}=v^{2} \frac{\partial}{\partial v} \frac{1}{v^{2}} \int_{-v}^{v} d x \int_{0}^{\infty} \frac{d k}{\pi} \operatorname{Re}\left\{\frac{\Pi_{L}(x)}{k^{2}+\Pi_{L}(x)}-\frac{v^{2}-x^{2}}{1-x^{2}} \frac{\Pi_{T}(x)}{k^{2}\left(1-x^{2}\right)+\Pi_{T}(x)}\right\} .
$$

We finally perform the integral over $k$,

$$
\frac{d_{\infty}}{C_{R} \alpha_{s}}=m_{D} v^{2} \frac{\partial}{\partial v} \frac{1}{v^{2}} \int_{0}^{v} d x \operatorname{Re}\left\{\sqrt{\hat{\Pi}_{L}(x)}-\frac{v^{2}-x^{2}}{\left(1-x^{2}\right)^{3 / 2}} \sqrt{\hat{\Pi}_{T}(x)}\right\},
$$

where

$$
\begin{aligned}
& \hat{\Pi}_{L}(x) \equiv \Pi_{L}(x) / m_{D}^{2}=1-\frac{x}{2} \log \left(\frac{x+1}{x-1}\right) \\
& \hat{\Pi}_{T}(x) \equiv \Pi_{T}(x) / m_{D}^{2}=\frac{x^{2}}{2}\left[1-\frac{x^{2}-1}{2 x} \log \left(\frac{x+1}{x-1}\right)\right] .
\end{aligned}
$$

The longitudinal contribution to $(\mathrm{B} .10)$ reads:

$$
\begin{gathered}
d_{\infty L}=-C_{R} \alpha_{s} m_{D} A_{L}(\gamma) \\
A_{L}(\gamma)=\frac{2}{v} \int_{0}^{v} d x \operatorname{Re} \sqrt{1-\frac{x}{2} \log \left|\frac{x+1}{x-1}\right|+\frac{i \pi x}{2}}-\operatorname{Re} \sqrt{1-\frac{v}{2} \log \left|\frac{v+1}{v-1}\right|+\frac{i \pi v}{2}} .
\end{gathered}
$$

We check numerically that $A_{L}(\gamma)$ is a smooth increasing function of the parton Lorentz factor $\gamma=1 / \sqrt{1-v^{2}}$, increasing very slowly above $\gamma=10$, and saturating when $\gamma \rightarrow \infty$ : $A_{L}(1)=1, A_{L}(10) \simeq 1.3, A_{L}(1000) \simeq 1.5, A_{L}(\infty) \simeq 1.814$.

For the transverse contribution to $B .10$ we find:

$$
\begin{gathered}
d_{\infty T}=-C_{R} \alpha_{s} m_{D} A_{T}(\gamma) \\
A_{T}(\gamma)=\frac{2}{v} \int_{0}^{v} d x \frac{x^{2}}{\left(1-x^{2}\right)^{3 / 2}} \operatorname{Re} \sqrt{\frac{x^{2}}{2}\left[1-\frac{x^{2}-1}{2 x} \log \left|\frac{x+1}{x-1}\right|\right]+\frac{i \pi x\left(x^{2}-1\right)}{4} .}
\end{gathered}
$$




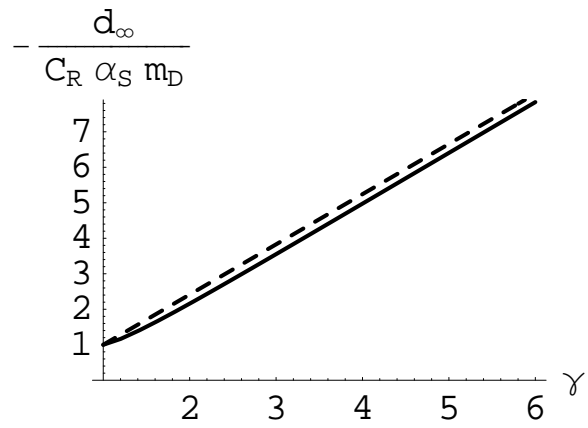

Figure 4: The exact function $-d_{\infty} /\left(C_{R} \alpha_{s} m_{D}\right)$ (full line) and its approximation $1+\sqrt{2}(\gamma-1)$ (dashed line), as a function of $\gamma=E / M$, see (B.17).

When $v \rightarrow 1$, a singularity at $x \rightarrow 1$ appears in the integrand of (B.15), and we easily derive the asymptotic behaviour $A_{T}(\gamma) \simeq \sqrt{2} \gamma$ when $\gamma \rightarrow \infty$, giving:

$$
d_{\infty T} \underset{v \rightarrow 1}{\simeq}-\sqrt{2} C_{R} \alpha_{s} m_{D} \gamma
$$

Numerically, $A_{T}(\gamma)$ (which satisfies $A_{T}(1)=0$ ) is quite well approximated by the linear form $\sqrt{2}(\gamma-1)$ for all values of $\gamma \geq 2$, with an accuracy of $25 \%$ for $\gamma=2$ and improving for increasing $\gamma$.

Adding (B.12) and (B.14) we obtain

$$
d_{\infty}=-C_{R} \alpha_{s} m_{D}\left(A_{L}(\gamma)+A_{T}(\gamma)\right) \simeq-C_{R} \alpha_{s} m_{D}(1+\sqrt{2}(\gamma-1)),
$$

where the latter approximation has the correct limits at $\gamma \rightarrow 1$ and $\gamma \rightarrow \infty$, and can be

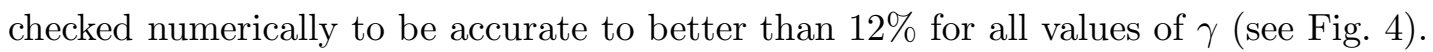

It is instructive to find what are the typical values of $k$ and $\theta$ which contribute to the leading behaviour $(\overline{B .16})$ of $d_{\infty}$. In $(\overline{B .9})$ the transverse contribution is dominated by $x=v \cos \theta \rightarrow 1$ when $v \rightarrow 1$, i.e. $\theta \ll 1$. Using $\Pi_{T}(x \rightarrow 1)=m_{D}^{2} / 2$, the leading part of (B.9) can be written as

$$
d_{\infty} \underset{v \rightarrow 1}{\simeq}-C_{R} \alpha_{s} \int_{0}^{1} \frac{d \theta^{2}}{\theta^{2}+1 / \gamma^{2}} \int_{0}^{\infty} \frac{d k}{\pi} \frac{m_{D}^{2}}{k^{2}\left(\theta^{2}+1 / \gamma^{2}\right)+m_{D}^{2} / 2} .
$$

The latter is easily checked to arise from $\theta \sim 1 / \gamma$ and $k \sim \gamma m_{D}$ (as well as to yield the result $(\overline{\mathrm{B} .16}))$.

\section{B.2 Contribution from radiation to $d_{\infty}$, in the $v \rightarrow 1$ limit}

In order to see how the bremsstrahlung induced by the initial acceleration of the parton affects the retardation effect, we single out in $d_{\infty}$, given by (B.17), the contribution from radiation. This contribution $W_{\infty}$ is obtained by subtracting (4.5) from (4.3), and then integrating over $k$ and $\theta$. Here we concentrate on the transverse contribution $W_{\infty} T$ which turns out to be dominant in the $v \rightarrow 1$ limit,

$$
\frac{W_{\infty} T}{C_{R} \alpha_{s}}=\int_{0}^{\infty} \frac{d k}{2 \pi} \int_{-1}^{1} d \cos \theta\left\{\frac{z_{T}(k) \sin ^{2} \theta}{\left(\cos \theta-\omega_{T}(k) /(k v)\right)^{2}}-\frac{\sin ^{2} \theta}{(\cos \theta-1 / v)^{2}}\right\} .
$$


When $v \rightarrow 1$, the above integral is dominated by the domain $\theta \ll 1, k \gg m_{D}$. For $k \gg m_{D}$ we have $\omega_{T}(k) \simeq k+m_{\infty}^{2} /(2 k)$, where $m_{\infty}=m_{D} / \sqrt{2}$ is the asymptotic gluon thermal mass, and $z_{T}(k) \simeq 1$ [15] can also be consistently used in (B.19). Approximating the integrand in $(\mathrm{B} .19)$ we obtain

$$
\frac{W_{\infty} T}{C_{R} \alpha_{s}} \underset{v \rightarrow 1}{\simeq}-\frac{2 m_{\infty}^{2}}{\pi} \int_{0}^{\infty} \frac{d k}{k^{2}} \int_{0}^{1} d \theta^{2} \theta^{2} \frac{\theta^{2}+\frac{1}{\gamma^{2}}+\frac{m_{\infty}^{2}}{2 k^{2}}}{\left(\theta^{2}+\frac{1}{\gamma^{2}}\right)^{2}\left(\theta^{2}+\frac{1}{\gamma^{2}}+\frac{m_{\infty}^{2}}{k^{2}}\right)^{2}} .
$$

In the ultrarelativistic $\gamma \rightarrow \infty$ limit, the typical values of $k$ and $\theta$ in the latter integral are $k \sim \gamma m_{\infty}$ and $\theta \sim 1 / \gamma$. The calculation is now straightforward and we obtain for the leading term:

$$
W_{\infty T} \underset{v \rightarrow 1}{\simeq}-C_{R} \alpha_{s} m_{D} \gamma / \sqrt{2} .
$$

This is exactly half of the full result for $d_{\infty}$, see (B.16) and (B.17).

For completeness we quote the result for the longitudinal contribution to $W_{\infty}$,

$$
W_{\infty L} \underset{v \rightarrow 1}{\simeq} \frac{2 C_{R} \alpha_{s} m_{D}}{3 \pi}(\log \gamma)^{3 / 2}
$$

which is indeed subleading compared to (B.21) when $v \rightarrow 1$.

\section{References}

[1] J. D. Bjorken, Fermilab preprint PUB-82/59-THY (1982).

[2] R. Baier, Y. L. Dokshitzer, S. Peigné and D. Schiff, Phys. Lett. B 345 (1995) 277 [hep-ph/9411409].

[3] M. H. Thoma and M. Gyulassy, Nucl. Phys. B 351 (1991) 491.

[4] E. Braaten and M. H. Thoma, Phys. Rev. D 44 (1991) 1298; 2625.

[5] A. Peshier and Y. L. Dokshitzer, private communications.

[6] R. Baier et al., Nucl. Phys. B 483 (1997) 291 [hep-ph/9607355].

[7] B. G. Zakharov, JETP Lett. 65 (1997) 615 [hep-ph/9704255].

[8] M. Gyulassy, P. Levai and I. Vitev, Nucl. Phys. B 594 (2001) 371 [nucl-th/0006010].

[9] M. G. Mustafa and M. H. Thoma, Acta Phys. Hung. A 22 (2005) 93 [hep-ph/0311168].

[10] A. K. Dutt-Mazumder, J. e. Alam, P. Roy and B. Sinha, Phys. Rev. D 71 (2005) 094016 [hep-ph/0411015].

[11] M. G. Mustafa, Phys. Rev. C 72 (2005) 014905 [hep-ph/0412402].

[12] K. Adcox et al. [PHENIX Collaboration], Phys. Rev. Lett. 88 (2002) 022301 [nucl-ex/0109003]; S. S. Adler et al. [PHENIX Collaboration], Phys. Rev. Lett. 91 (2003) 072301 [nucl-ex/0304022].

[13] C. Adler et al. [STAR Collaboration], Phys. Rev. Lett. 90 (2003) 082302 [nucl-ex/0210033];

J. Adams et al. [STAR Collaboration], Phys. Rev. Lett. 91 (2003) 172302 [nucl-ex/0305015]. 
[14] R. D. Pisarski, Physica A 158 (1989) 146. E. Braaten and R. D. Pisarski, Phys. Rev. Lett. 64 (1990) 1338; Nucl. Phys. B 337 (1990) 569; Nucl. Phys. B 339 (1990) 310.

[15] J. P. Blaizot and E. Iancu, Phys. Rept. 359 (2002) 355 [hep-ph/0101103].

[16] S. Ichimaru, 'Basic Principles of Plasma Physics', Benjamin, Inc., Advanced Book Program, 1973.

[17] V. V. Klimov, Sov. Phys. JETP 55 (1982) 199 [Zh. Eksp. Teor. Fiz. 82 (1982) 336].

[18] H. A. Weldon, Phys. Rev. D 26 (1982) 1394.

[19] A. Peshier, K. Schertler and M. H. Thoma, Annals Phys. 266 (1998) 162 [hep-ph/9708434].

[20] J. P. Blaizot, A. Ipp and A. Rebhan, hep-ph/0508317.

[21] M. Djordjevic and M. Gyulassy, Phys. Rev. C 68 (2003) 034914 [nucl-th/0305062].

[22] F. Wang [STAR Collaboration], J. Phys. G 30 (2004) S1299 [nucl-ex/0404010], see also nucl-ex/0508021; S. S. Adler et al. [PHENIX Collaboration], nucl-ex/0507004.

[23] D. d'Enterria, nucl-ex/0504001.

[24] V. S. Pantuev, hep-ph/0506095 and hep-ph/0509207. 